Erschienen als:

Keller, Felix. 2007. Figuren des Publikums. Politischer und diagrammatischer Raum.

In: Irma Schneider und Isabell Otto (Hrsg.).

Formationen der Mediennutzung II.

Strategien der Verdatung. Bielefeld.

Transcript. S. 153-170.

\section{Figuren des Publikums. Politischer und DIAGRAMMATISCHER RAUM}

\section{FELIX KELLER}

Die Idee des politischen Publikums, so zentral sic für politische Kommunikation und Herrschaft auch sein mag, geht einher mit eine Paradoxie: Die prinzipielle Offenheit des Zugangs zu politischen Aushandlungsprozessen, Rechtfertigung aller demokratischen Regimes, lässt sich aktuell nur über die faktische Einschränkung des Zugangs zu ihnen verwirklichen. ${ }^{1}$ Die gleichzeitige Partizipation Aller bei identischen Rechten und Zugangsmöglichkeiten führte auf jeden Fall unweigerlich zum Zusammenbruch bestehender politischer Institutionen. Diese Paradoxie mündet in viel diskutierte Mechanismen politischer Inklusion und Exklusion, ${ }^{2}$ doch sie verweist auch auf wissens- und medientechnische Voraussetzungen politischer Demokratien, denen sich dieser Beitrag widmet.

Im Spannungsfeld der Idee der Demokratie und der Möglichkeit ihrer Verwirklichung, so das Argument, entsteht eine Dynamik der Repräsentation des >politischen Publikums<, die sich die aktuellsten Wissenstechniken aneignet, um das Publikum des Staates doch in den politischen Kommunikationskreislauf einzubeziehen, es in der politischen Ordnung

1 Vgl. zur neueren Diskussion Jacques Rancières Arbeit mit dem Titel: Das Unvernehmen (Jacques Rancière: Das Unvernehmen. Politik und Philosophie, Frankfurt/Main: Suhrkamp 1995). Rancière zeigt, dass die politischen Institutionen eigentlich auf einen »nicht-teilhabenden Teil« angewiesen sind, um sich selbst als politisch zu konstituieren. In wachen Zeiten ist die Repräsentation des Souveräns in den Institutionen der Demokratie deshalb gerade der Kritikpunkt jener, welche die demokratische Bewegung verteidigen (S. 106f.). Andere Richtungen, allen voran jene Habermas', sehen ein nicht gemindertes »Potential der Selbsttransformation« des bürgerlichen Publikums, i.e. der »bürgerlichen Öffentlichkeit«, wie Habermas im Vorwort zu einer Neuauflage des Strukturwandels der Öffentlichkeit schreibt (Jürgen Habermas: Strukturwandel der Öffentlichkeit. Untersuchungen zu einer Kategorie der bürgerlichen Gesellschaft [1962]. Mit einem Vorwort zur Neuauflage 1990, Frankfurt/Main: Suhrkamp 1990, S. 20f.).

2 Vgl. zur neueren Diskussion der Begrifflichkeit Niklas Luhmann: »Jenseits von Barbarei«, in: ders., Gesellschaftsstruktur und Semantik. Studien zur Wissenssoziologie der modernen Gesellschaft, Bd. 4, Frankfurt/Main: Suhrkamp 1995, S. 138-150. 
zu repräsentieren. Auf diese Weise entstehen immer neue Figuren des Publikums: keineswegs stabile Figuren und auch in sich selbst widersprüchliche Figuren. Eine der folgenreichsten Innovationen zur Inklusion - und gleichzeitig Kreation - eines politischen Publikums stellt hierbei die Einführung der Meinungsforschung dar, die, mit Ansätzen zu Beginn der 20er Jahre des letzten Jahrhunderts, ${ }^{3}$ sich in den 30er Jahren in den USA unversehens durchzusetzen begann und eine umfassende Verdatung der ’öffentlichen Meinung ‘ angesichts neuer medientechnischer Realitäten versprach: eine Strategie, die angesprochenen Paradoxien anzugehen, die sich noch immer auf einem ungebrochenen Siegeszug befindet und verspricht, selbst die Nicht-Teilnehmenden, die sonst nicht Repräsentierten, in ihren Untersuchungen einschließen zu können. Dieser Artikel richtet das Augenmerk auf den Versuch, die potenziell politische Bevölkerung des Staates zumindest wissenstechnisch zu integrieren, in einem bildlichen Raum sichtbar zu machen. ${ }^{4}$

\section{Ein seltsames politisches Porträt}

Die angesprochene Paradoxie demokratischer Inklusion und politischer Repräsentation lässt sich anhand eines eigentümlichen Gemäldes des britischen Porträt- und Historienmalers George Hayter nachvollziehen. Im Jahre 1833 präsentierte Sir George Hayter ${ }^{5}$ ein Gemälde mit dem Namen The reformed House of Commons, das »Parlament « des britischen Königreichs darstellend. Auf den ersten Blick wenigstens scheint dieses Bild wenig spektakulär. Ungeachtet seiner immensen Größe $(5,4 \mathrm{~m}$ auf $3,5 \mathrm{~m})$ ist es von einer ästhetischen Ruhe gekennzeichnet, welche die Komposition nahe an die Grenze erstarrter Gleichförmigkeit rückt: Der Aufbau ist

3 Vgl. bezüglich der Befragung Louis L. Thurstone: „Attitudes Can Be Measured «, in: American Journal of Sociology 4 (1929), S. 529-554; bezüglich der Entwicklung statistischer Stichproben in den USA der 20er Jahren vgl. Alain Desrosières: ")Three Studies on the History of Sampling Surveys: Norway, Russia-USR, United-States«, in: Science in Context 15 (2002), S. 377-383, hier $380 \mathrm{f}$.

4 Vgl. zur weitergehenden Analyse der Antinomien der Meinungsforschung Felix Keller: Archäologie der Meinungsforschung. Mathematik und die Erzählbarkeit des Politischen, Konstanz: UVK 2001.

5 Hayter gilt als wichtiger, wenn auch nicht überragender britischer Maler der ersten Hälfte des 19. Jahrhunderts (vgl. Richard Ormond: »The Hayters«, in: The Burlington Magazine 124 (1982), H. 956, S. 711-712). Seine Gemälde folgen den Konventionen der Zeit, wie sie darin auch in gewisse Weise stilbildend sind. Vgl. Frederick Voss: Webster Replying to Hayne. George Healy and the Economics of History Painting, in: American Art 15 (2001), S. 34-53, hier S. 38. streng symmetrisch, beinahe geometrisch. Zwei Reihen von sitzenden und im Vordergrund stehenden Parlamentariern fügen sich spiegelbildlich in den Fluchtpunkt dreier heller Fenster. Auf den Balkonen, The Singers Gallery, sind ebenfalls Figuren erkenntlich: das die politische Aushandlung verfolgende Publikum. Dort finden sich leere Sitze, es gibt genügend Platz für die räumliche Integration der an den Verhandlungen Interessierten. Einen anderen Eindruck hinterlässt die Reihung der Parlamentarie auf ihren Sitzen: Sie reihen sich gedrängt, eigentümlich in ihrer Gestalt sichtbar, aber doch streng geordnet.

Hayters Gemälde entstand in einer Zeit, die revolutionäre Züge trug: Die Industrialisierung hatte nicht nur für soziale Unrast gesorgt, sondern auch das System der politischen Repräsentation des Britischen Königreichs aus den Fugen gebracht. Die Reform Bill des Jahres 1832, deren parlamentarisches Ergebnis Hayter zu verbildlichen suchte, stellte einen Versuch dar, die politische Repräsentation des Britischen Königreichs zu rationalisieren. Dies geschah im Akt von 1832 vornehmlich dadurch, dass das Gleichgewicht zwischen den industrialisierten Regionen und Städten und den vornehmlich vom Adel dominierten Landstrichen wiederhergestellt und die Wählerbasis beinahe verdoppelt wurde. Die Situation zeigte sich ähnlich wie im vorrevolutionären Frankreich: Die immensen Klassengegensätze berücksichtigte diese Reform Bill nicht, nur ungefähr 4 Prozent der Bevölkerung waren wahlberechtigt. ${ }^{6}$ Ungeachtet ihrer politischen Bedeutung war die Reform Bill ein Versuch, die politische Repräsentation noch innerhalb der bestehenden Matrix politischer Machtverhältnisse mathematisch zu adjustieren und dadurch über größere Inklusion Legitimität zu schaffen, respektive die Unruhen zu besänftigen.

Inwiefern stellt Hayters Gemälde hinsichtlich des angesprochenen Paradoxes eine Besonderheit dar? Hayter begann dieses Gemälde des neu gebildeten House of Commons 1832 und arbeitete ganze zehn Jahre daran. Die Arbeit dauerte so lange, weil Hayter versuchte, möglichst viele, ja alle der Parlamentarier einzeln zu porträtieren, ihnen ein unverwechselbares Profil zu geben. Die National Portrait Gallery, in der sich das Gemälde befindet, vermutet, dass es das Porträt mit den meisten Porträtierten darstellt. ${ }^{7}$ Freilich, die Zeit der Erstellung der Porträts der Parliament Members dauerte länger als der politische Zyklus des Wechsels von Parlamentsmitgliedern, die zeitliche Fluktuation ließ sich nicht in die synchrone Repräsentation einbinden. Doch weitere Probleme stellten sich Hayter: Auf welche Weise hätte er sämtliche 658 Parlamentsmitglieder im Gemälde

6 Vgl. zur Darstellung und Interpretation dieser parlamentarischen Reformprozesse Sean Lang: Parliamentary Reform 1783-1867, London: Routledge 2004.

7 »Portrait with most sitters? «, fragt sich die National Portrait Gallery (vgl. http://www.npg.org.uk/live/unusual.asp vom 3.8.2007). 
darstellen können? Die erste Schwierigkeit rührt hier schon allein daher, dass der Ort, an dem das Parlament residiert, St. Stephen's Chapel, lediglich 427 Parlamentsmitgliedern erlaubte Platz zu nehmen. Dazu kamen die technische Schwierigkeit der Größe des Gemäldes und die damit verbundene Frage, mit welchem Ausmaß und wie viele Parlamentarier sich auf einer gegebenen Fläche überhaupt als Individuen erkennbar darstellen lassen. Hayter sah sich so gezwungen, lediglich 327 Parlamentsmitglieder zu porträtieren. Freilich, wie ließ sich damit noch das eigentlich Besondere des neuen Parlaments darstellen, das sich gerade dadurch auszeichnete, dass es die Wahlrechte ausweitete, mathematisch neu proportionalisierte, und so die parlamentarische Repräsentation verbreiterte und den Delegierten einen neuen Raum des politischen Räsonierens zur Verfügung stellte? Hayter entschied sich, die Zahl der Parlamentsmitglieder proportional zur der Parteistärke auf dem Bild erscheinen zu lassen. Nach welchen Kriterien er Parlamentsmitglieder porträtierte und andere schlicht ignorierte, um den proportionalen Anteil erhalten zu können, bleibt der Gegenwart verschlossen.

Klar ist aber, dass Hayter sich damit in einen unüberbrilickbaren Widerspruch verstrickte: Die Idee der politischen Subjektivität, deren symbolische Darstellungsweise nur das Porträt sein konnte, und die mathematische Logik der Repräsentation lassen sich nicht auf einen Nenner bringen. ${ }^{8}$ Hayter hatte entsprechend wenig Glück mit seinem auf den zweiten Blick durchaus einmaligen Gemälde; es blieb über Jahre hinaus unverkäuflich, ungeachtet seiner großen Bemühungen. Die Reform Bill und das Parlament, das sie hervorbrachte, waren, gerade wegen ihrer Widersprüche, bereits Geschichte. Die neuen Verhältnisse hatten sich geweigert, sich in einem Raum der Sichtbarkeit darstellen zu lassen.

Ungeachtet - oder gerade wegen - dieses unglücklichen Schicksals ist dieses Bild bemerkenswert, weil es zwei verschiedene Ordnungen der Konstitution einer Republik zu harmonisieren versuchte, die in gewissem Sinne unverträglich sind, doch im Modus des Politischen sich beständig produzieren: die Subjektivierung politischer Meinung (als politische Position eines sich selbst bewussten Staatsbürgers, der in der Öffentlichkeit räsoniert und damit Öffentlichkeit gleichzeitig hervorbringt,) und die mathematische Logik der Repräsentation des Souveräns und ihrer Vertreter selbst, welche die Politik über ihre Stimmen (im zweifachen Sinne des

8 Ohne Zweifel ließen sich die Widersprüche dieses Gemäldes (wie auch die späteren Widersprüche der Meinungsforschung) in der Logik des Wissens verstehen, das, wie Foucault (Michel Foucault: Die Ordnung der Dinge, Frankfurt/Main: Suhrkamp 1991) zeigte, mit der schwindenden Geltungskraft des klassischen Tableaus der Repräsentation einhergeht, die Vorstellung des Menschen als Subjekt hervortreten und die mathematische Ordnung sich verselbstständigen lässt
Wortes) der demokratischen Idee nach gestalten. Was hier für Hayter eine Unmöglichkeit war, diese beiden Logiken im Bild der St. Stephen's Chapel ineinanderzuführen, stellt indes eine Herausforderung dar, die immer wieder zu neuen technologischen Innovationen führte. Kumulationspunkt dieser Aporie ist die massive Mathematisierung und Verdatung politischer Stimmen und politischer Repräsentation über die Verfahren der Meinungsforschung.

\section{Die Imagination stattfindender Politik}

Offensichtlich folgte Hayter einer ganz bestimmten Vorstellung, auf welche Weise das politische Publikum und die demokratische Auseinandersetzung sich gestaltet, auf welche Weise republikanische Politik stattfindet. Was auf dem Gemälde dargestellt ist, könnte als eine Art , Verbildlichung ‘ einer Vorstellung des Souveräns bezeichnet werden, die mit einer ganz spezifischen `Ästhetik $\prec$, oder `Ästhetik der Demokratien einhergeht. Erst mit einer Plausibilisierung über dieses Bild der Demokratien vermochte die Meinungsforschung sich in der Öffentlichkeit als ein sinnvolles mathematisches Darstellungsverfahren darzustellen, das die Probleme der politischen Inklusion in großen Gesellschaften zu lösen vermag.

Diese Ästhetik der Demokratien gründet im Bild einer politischen Ordnung, die getragen wird und erzeugt wird von Subjekten, die zwar in sich selbst eigenartig sind, doch in einem klar identifizierbaren Bezug zueinander stehen, in einem Bezugssystem von Positionen und Argumenten, in einem System von >Angesicht zu Angesicht<, ohne Hinterbühne ${ }^{9}$ und Konstitutionsprobleme. In diesem Sinne wird stattfindende Politik wahrnehmbar bzw. über die Darstellungsweise wahrnehmbar gemacht oder eben: ästhetisiert. Diese Ästhetik scheint tief in das politische Bewusstsein der Moderne eingelassen, sie sucht sich die verschiedensten historischen Orte, in denen sie nicht nur ein ästhetisches Ideal darstellt, sondern de facto auch funktioniert - und gerade die Ästhetik verhilft aus der Paradoxie der Inklusion, wie sich unschwer anhand der Diskussion des gleichsam ersten >Imaginationsortes demokratischer Ordnung zeigen lässt: der griechischen Polis, die als physischer Ort stattfindender Politik auch die Züge des Bildhaften trägt. Das Modell der Polis-Öffentlichkeit "strahlt« in seiner normativen Kraft bis in die Gegenwart, ${ }^{10}$ wobei damit

9 Vgl. zu diesem für die örtliche Kommunikation essenziellen Konstrukt, das im Phänomen der totalen Sichtbarkeit ausgeblendet wird: Erving Goffman: Wir alle spielen Theater. Selbstdarstellung im Alltag [1959], München, Zürich: Piper 1996, S. 104.

10 Als Beispiele seien nur zu nennen: James Bryce: Moderne Demokratien, Bd. 1, München: Drei Masken 1923; Hannah Arendt: Vita Activa oder vom täti- 
weniger die gesellschaftliche Ordnung gemeint ist als das, was Habermas als »ideologisches Muster« bezeichnet: ${ }^{11}$ Das Augenmerk richtet sich auf diese normative Kraft und weniger auf die Konstitutionsbedingungen der Norm selbst. Doch die Artikulation der Norm kommt nicht gänzlich ohne die Imagination ihrer Realisierung aus: Die historischen Ausführungen zur griechischen Polis nehmen damit in der politischen Theorie die Qualität und Funktion an, welche das Gemälde Hayters in visueller Hinsicht hatte. Die Erzählungen der politischen Theorie zeigen den historischen Ort der Politik in einem geschlossenen Raum der perfekten Sichtbarkeit.

Explizit kommt dieses Element der Sichtbarkeit als Voraussetzung für stattfindende Politik bei Hannah Arendt zum Zuge: Der öffentliche Raum als Ort der Politik bedeutet für Arendt primär, »daß alles, was vor der Allgemeinheit erscheint, für jedermann sichtbar und hörbar ist, wodurch ihm die höchstmögliche Öffentlichkeit zukommt «. ${ }^{12}$ Die Polis ist rückschauend aber nicht nur für Arendt, sondern auch für die historisch operierende Soziologie konstituiert als umfassende, wenn nicht totale Sichtbarkeit. ${ }^{13}$ Wird man sich indes der Tatsache der \Örtlichkeit< des Geschehens bewusst, der Konstitution aus Fleisch und Stein, um mit Richard Sennett zu sprechen, ${ }^{14}$ so zeigt sich, dass die Konkurrenz und Inszenierung der Besten gegenüber den anderen Gleichen, die Arendt so begeisterte, auf Konstitutionsbedingungen angewiesen sind, die selbst in dieser Norm nicht enthalten sind: ein System der Präsenz, Adressierbarkeit und der Sichtbarkeit der Anderen, ohne das die Konkurrenz und Profilierung der Besten so nicht funktionierte. Wenn das konstituierende Element der Sichtbarkeit dieser republikanischen Ordnung nicht selbst enthalten ist, so kann entsprechend die weitere Geschichte politischer Gesellschaften nur als eine Zerfallsgeschichte dargestellt werden, die bei Arendt in eine Geringschätzung der Massengesellschaft mündet. Mit anderen Worten: Die Kritik des Bestehenden verhilft aus der Paradoxie der Inklusion Aller.

gen Leben, München: Pieper 1981; Habermas: Strukturwandel der Öffentlichkeit (wie Anm. 1); Richard Sennett: Fleisch und Stein. Der Körper und die lichkeit (wie Amotlichen Zivilisation, Frankfurt/Main: Suhrkamp 1997; CorStadt in der westlichen Zivilisation, Frankfurt/Main: Sump 1997, Cornelius Castoriadis: »La polis grecque et la démocratie«, in: ders., Domaines de l'homme. Les carrefours du labyrinthe II, Paris: Seuil 1986, S. 261-306.

1 Habermas: Strukturwandel der Öffentlichkeit (wie Anm. 1), S. $57 \mathrm{f}$.

12 Arendt: Vita Activa (wie Anm. 10), S. 43.

13 Vgl. Sennett: Fleisch und Stein (wie Anm. 10), S. xi. Vgl. auch das Kapitel Die Polis als Raum der Sichtbarkeit«, in: Jakob Stefan Seitz, Hannah Arendts Kritik der politisch-philosophischen Tradition: unter Einbeziehung der französischen Literatur zu Hannah Arendt, München: Utz 2002, S. $138 \mathrm{ff}$.

14 Vgl. Sennett: Fleisch und Stein (wie Anm. 10).

\section{Der geometrisierte Souverän}

Gerade gegenteilig existieren vice versa auch Versuche, angesichts veränderter gesellschaftlicher Verhältnisse die konstitutiven Bedingungen dieses sichtbaren Raums über technologische Mittel schlicht nachzubauen, in der Hoffnung, das Phänomen des durch Sprache und Argumentation gefüllten politischen Raums stelle sich ein, wenn sich das Publikum nunmehr über die verschiedenen Techniken der Wissensschaffung erneut selbst zu schaffen weiß. Als ein wirkungsmächtiges Instrument zur Beruhigung der Paradoxie erweist sich dabei der Versuch - er kommt auch bei Hayters Gemälde zum Ausdruck -, die politische Ordnung zu mathematisieren bzw. über statistische Verhältnisse zur Wirklichkeit zu erheben. Der Widerstand, der diesem Lösungsversuch seitens der an der Polis gewonnenen Idee entgegenschlägt, zeigt gerade, inwiefern er eine Alternative zur Artikulation des normativen Horizonts darstellt. Dieser Widerstand lässt sich wiederum exemplarisch an Arendts Werk nachzeichnen.

Das Phänomen des technischen Raums ist für Arendt untrennbar mit dem Auftauchen von Gesellschaft verbunden, die wiederum in der "schieren Addierung vieler Familien « gründet, ${ }^{15}$ die im Konstrukt der Gesellschaft absorbiert werden wie später andere Formationen, etwa Klassen und Gruppierungen. Gesellschaft findet ihre geklärte Form in der »Massengesellschaft«, als »Sieg der Gesellschaft überhaupt.« Vor der Gesellschaft sind alle gleich, im Sinne einer Egalität des Ausgesetztseins gegenüber sozialen Tatsachen. Diese Egalität ist eine andere als die Gleichheit als Voraussetzung der Profilierung der Überdurchschnittlichen in der griechischen Polis, oder, anders gesagt: etwas anderes als die Subjektivität der Individuen. Die Wissenschaft, die dem »Entstehen der Gesellschaft auf dem Fuße folgt«, ist für Arendt die Statistik. ${ }^{16}$ Mit anderen Worten gesagt: Die Statistik ist jene symbolische Form, in der sich die Gesellschaft als Tatsache selbst äußert. Statistik hat es zwar schon länger gegeben, aber die Wissenschaftlichkeit dieser Techniken ergibt sich erst, indem Gesellschaft selbst schon Formen aufweist, die sich einheitlich systematisieren lassen. Die Statistik, die großen Zahlen als symbolische Formen der Gesellschaft, sind als solche der Form der Gesellschaft für Arendt durchaus adäquat. Das Problem liegt für Arendt darin, dass bei der Behandlung dieser Zahlen als gesellschaftliche Realität in Vergessenheit gerät, was ihre Bedeutung, ihre Geltung überhaupt erst geschaffen hat: nämlich der Raum des Politischen und die Ordnung, die als Singularität eben gerade nicht ein Ereignis in einer Serie der großen Zahl waren, sondern für Arendt Ergebnis der Tätigkeit von Subjekten. Freilich ist es ge-

15 Arendt: Vita Activa (wie Anm. 10), S. 40ff.

16 Ebd., S. 42. 
rade die große Zahl selbst, das Anwachsen der Bevölkerung, das dafür sorgt, dass der Raum der großen Zahl, die Massengesellschaft, zur allein bestimmenden Größe wird und nicht mehr das politische Handeln der Subjekte:

»Aus der unbestreitbaren Gültigkeit statistischer Gesetze im Bereich großer Zahlen folgt leider für die Welt, in der wir leben, nur, daß jede Zunahme der Bevölkerung diesen Gesetzen eine erhöhte Geltung verleiht, der gegenüber dic $>$ Abweichungen immer gegenstandsloser werden. Politisch gesprochen heißt das: je größer die Bevölkerung der jeweiligen politisch konstituierten Gemeinschaften anwächst, desto wahrscheinlicher ist es, daß das Gesellschaftliche und nicht das politische Element den Vorrang innerhalb des öffentlichen Bereiches erhält. « ${ }^{17}$

Die große Zahl, die Zahl der Bevölkerung, die schon in der Polis die Sorge um die Begrenzung der Vielen evoziert hat, ${ }^{18}$ zeigt damit bei Arendt eine eigentümliche doppelte Bedeutung. Sie ist gleichzeitig Ausdruck der Abwesenheit des öffentlichen Raums wie auch die Ursache seines Verschwindens: Letztlich trifft sie die Realität nicht, aber sie ist doch entscheidend real für die Gesellschaft. Es ist dieses >leider`, das Arendt von einer nüchternen theoretischen Beschreibung der Genese des gesellschaftlichen Raums als einem statischen bzw. diagrammatischen Raum trennt.

Freilich, in nichts Geringerem als in der amerikanischen Verfassung, dem Konstitutionsort einer modernen Gesellschaft schlechthin, komm die unhintergehbare Bedeutung der großen Zahl für die Bildung moderner Gesellschaften zum Ausdruck. Die amerikanische Verfassung ist geradezu ein Paradebeispiel für den Versuch, die Paradoxien der politischen Repräsentation eines sich bildenden Staatswesens über mathematische Geometrisierung und Verdatung zu lösen. Bereits der Artikel 1, Abschnitt 2, berichtet davon

»Niemand kann Abgeordneter werden, der nicht das Alter von 25 Jahren erreich hat, sieben Jahre Bürger der Vereinigten Staaten gewesen und zur Zeit seiner Wahl Einwohner desjenigen Staates ist, in dem er gewählt wird. Die Abgeordnetenmandate und die direkten Steuern werden auf die einzelnen Staaten, die diesem Bund angeschlossen sind, im Verhältnis zu ihrer Einwohnerzahl verteilt; diese wird ermittelt, indem zur Gesamtzahl der freien Personen, einschließlich der in einem befristeten Dienstverhältnis stehenden, jedoch ausschließlich der

17 Ebd., S. 43.

18 Vgl. etwa Aristoteles: Politik. Schriften zur Staatstheorie. Übersetzt und hg. v. Franz F. Schwarz, Stuttgart: Reclam 1989, S. 1326b u. S. 1327b; Plato: Nomoi, Göttingen: Vandenhoeck \& Ruprecht 1994, S. 745c, 737e, 785b. nicht besteuerten Indianer, drei Fünftel der Gesamtzahl aller übrigen Personen hinzugezählt werden. ${ }^{19}$

Die Verfassung fährt in ähnlicher Weise fort mit der Bestimmung von Senatoren und schlussendlich des Präsidenten. Der amerikanische Souverän schafft sich als eine geometrische Ordnung die Voraussetzung und das Bestimmungsmaß des Chors der politischen Stimmen. Diese Schrift is insofern bemerkenswert, als sie nicht die Frage des adäquaten Messens oder Abbildens behandelt, sondern die Schaffung eines Raumes über ein Schriftstück: Der Raum, der mit diesem Dokument geschaffen wird, bildet den Souverän nicht $\mathrm{ab}$, er ist der Souverän. Zudem zeigt sich auch, wie essenziell die Rückbeziehung auf die Ordnung der Körper und der >Stimmen` (im mehrfachen Sinne) ist. Ungeachtet der für demokratische Ordnung unhinterfragbaren Bedeutung der Meinungsfreiheit und ihrer medialen Vermittlung ist die Ordnung der lebenden Körper, des Scheidens jener, die dazugehören, von jenen, die nicht dazugehören, entscheidend: Sie ist gleichsam die Verankerung der Republik und ihrer demokratischen Ordnung in der Körperlichkeit der Staatsmitglieder, die selbst nie über Delegationsverhältnisse aufgehoben werden kann. ${ }^{20}$ Doch einer technischen Lösung der Verwirklichung der demokratischen Idee ist damit keineswegs der Weg gewiesen. Das Ringen um die Paradoxien des Ausschlusses und der Integration wird bereits in der amerikanischen Verfassung deutlich, der Versuch, den Souverän über seine Grenzen zu definieren, mit teils merkwürdig anmutenden Wendungen - »nicht besteuerte Indianer«, Berücksichtigung der »Personen im befristeten Dienstverhältnis« usf. In einem revolutionären $\mathrm{Akt}$ des Bildens eines Souveräns wird dieser nun in einer mathematischen Form gleichzeitig stillgelegt.

19 Verfassung der Vereinigten Staaten von Amerika, 17.9.1787. Eine deutsche Fassung findet sich auf der Webseite der amerikanischen Botschaft in Deutschland unter http://usa.usembassy.de/etexts/gov/gov-constitutiond.pdf vom 16.7.2007. Die verschiedenen Revisionen zeigen, inwiefern die Zulassungskriterien die eigentlich umkämpften Grenzen darstellen.

20 Vgl. hierzu Horst Bredekamp: Thomas Hobbes. Der Leviathan. Das Urbild des modernen Staates und seine Gegenbilder, 1651-2001, Berlin: Akadamie Verlag 2003. 


\section{Das politische Publikum und der Raum der Sichtbarkeit}

Die Paradoxie zwischen Subjektivierung und Integration der Vielen kann über die Schaffung eines technisch-statistischen Raumes ebenso wenig gelöst werden ${ }^{21}$ wie über die Artikulation eines idealen Horizonts der Politik, der das Bestehende als das Falsche brandmarkt. Immerhin lassen sich aufgrund eines Nachzeichnens der Neuerschaffung von Figuren des politischen Publikums die Strategien erkennen, die die eingangs erwähnte Unruhe und Paradoxie demokratischer Aushandlungsprozesse - zumindest temporär - stillzulegen vermögen. Dieser Prozess der Geometrisierung, der Schaffung eines neuen Raumes über neue Bestimmungsmöglichkeiten, ist soziologisch durchaus nachvollziehbar: nämlich als ein Lösungsversuch der Inklusionsproblematik der Demokratie, als ein umfassender Prozess der Transformation gesellschaftlicher Wahrnehmung und gesellschaftlichen Wissens, der die Entstehung moderner Gesellschaften begleitet und mit dem zusammenhängt, was gemeinhin unter $>$ Abspaltung des Raumes vom Ort verstanden wird. Prägnant hat diesen Transformationsprozess Anthony Giddens beschrieben. ${ }^{22}$ Unter >Ort< versteht Giddens die Vorstellung eines lokalen Schauplatzes; damit gemeint ist das Einhergehen physischer Umgebungsbedingungen mit gesellschaftlichen Tätigkeiten. In vormodernen Zeiten, so Giddens, fallen Raum und Ort weitgehend zusammen über die Bedingungen von Anwesenheit (der Personen). Die »räumlichen Dimensionen des gesellschaftlichen Lebens realisieren sich in einem Schauplatz. ${ }^{23}$ Das heißt nun wiederum nicht, dass Schauplätze der Gesellschaft in der Moderne verschwinden würden, sie werden nur noch nicht durch Anwesenheit konstituiert, sondern durch abstrakte Momente jenseits der unmittelbar physischen Sichtbarkeit. Entscheidend für die Loslösung des Raums vom Ort ist die Kreation symbolischer Medien, welche die Kommunikation zwischen abstrakten Instanzen (beispielsweise Geldwert) und Schauplätzen des Geschehens (konkrete Märkte) übernehmen. Die Standardisierung von abstrakten Dimensionen wie Raum und

21 Diese wissenstheoretische Spannung, die selbst unauflösbar erscheint, zeigt sich notabene auch in den Sozialwissenschaften, die in der Kluft zwischen subjektivierenden und objektivierenden Verfahren keinen stabilen Ort finden. Vgl. dazu Hubert L. Dreyfus/Paul Rabinow: Michel Foucault. Jenseits von Strukturalismus und Hermeneutik, Frankfurt/Main: Athenäum 1987.

22 Vgl. Anthony Giddens: Konsequenzen der Moderne, Frankfurt/Main: Suhrkamp 1995, S. 29ff. Giddens meint mit >Abspaltung des Raumes vom Ort<, dass - vornehmlich indiziert durch das Hervortreten der Zeitlichkeit aus der Ordnung als abstrakte, aber wirklich gemachte Kontinuität - der Ort selbst an Bedeutung verliert, ein ,Wenn nicht mehr notwendigerweise mit einem ,Wor gekoppelt ist. Die zeitübergreifende Koordinierung entleert den Raum.

23 Ebd., S. 30ff.
Zeit sowie symbolischer Medien, die innerhalb dieser Dimensionen fluktuieren, bedeutet, dass ein neues System wirkungsmächtiger Bezüge über die Spezifizität eines Ortes hinaus geschaffen wird, - was Giddens eben als Dislozierung und Schaffung von $>$ Raum $<$ versteht. Während sich diese Bewegung beispielsweise hinsichtlich der Märkte vom konkreten StadtOrt hin zu abstrakten ökonomischen Räumen (Märkten) über das abstrakte Medium Geld relativ gut nachzeichnen lässt, stellt sich die Frage, auf welche Weise der Raum des Politischen, der den Souverän konstituiert, diesen Prozess der Dislozierung verarbeitet.

Giddens nennt als Abstraktionsform diesbezüglich schlicht »Medien der politischen Legitimität« ${ }^{24}$ Doch ist hinsichtlich der Erzeugung eines Raums stattfindender Souveränität jenseits der Orte des Politischen auch die Bedeutung der Statistik zu nennen, welche zunächst, ganz im Sinne der Episteme der Klassik, wie sie Foucault identifiziert hat ${ }^{25}$ die Teile des Souveräns (seine Taxonomia) jenseits des reellen Ortes zu einem klassischen Tableau zusammenfügt, das ihn auf einer anderen logischen Ebene wieder konstituiert, im Wortsinn, den Foucault gebraucht: klassifikatorische Entität und Bild zugleich. ${ }^{26}$

Die amerikanische Verfassung, als Paradigma eines modernen Staates, bildet gleichsam den konstitutiven Rahmen, damit eine statistische Ordnung sich über die unübersichtliche Vielheit der Dinge legen kann. Für Alain Desrosière entwickelt sich in seiner Untersuchung zur Politik der großen Zahlen hingegen ein inhärenter Zusammenhang zwischen dem röffentlichen Raum und statistischen Evidenzen, in dem die Daten einer Gesellschaft debattiert werden. Der >öffentliche Raum r ist so mitnichten bloß Metapher im analytischen Diskurs, sondern ein historisch und technisch strukturierter und >eingegrenzter Raum<, der über die >Abstraktionsweise $<$ der statistischen Formen gleichsam als System von Relationen, von Distanzen und Äquivalenzen Wirklichkeit gewinnt. Dieser Zusam-

24 Ebd., S. 34

25 Vgl. Foucault: Die Ordnung der Dinge (wie Anm. 8).

26 Evidenterweise müssten hier auch die Medien genannt werden, va. die Bedeutung der Zeitung hinsichtlich der Formierung einer bürgerlichen Öffentlichkeit (vgl. den Artikel »Öffentlichkeit«, in: Otto Brunner/Werner Conze/ Reinhart Koselleck (Hg.), Geschichtliche Grundbegriffe: historisches Lexikon zur politisch-sozialen Sprache in Deutschland, Bd. 4, Stuttgart: Schwabe 1978, S. 413-467) und sozialer Bewegungen mit ihren Aktionsformen (Friedhelm Neidhardt: „Öffentlichkeit, öffentliche Meinung, soziale Bewegung«, in: ders. (Hg.), Öffentlichkeit, öffentliche Meinung, soziale Bewegung. Sonderheft 34 der Kölner Zeitschrift für Soziologie und Sozialpsychologie, Opladen: Westdeutscher Verlag 1994, S. 7-41). Doch hier geht es zunächst um die basale Frage, auf welche Weise überhaupt die Grenzen des Raums konstituiert werden, innerhalb dessen kommuniziert wird bzw. Kommunikationen zu Herrschaft transformiert werden. 
menhang, obwohl Desrosière seinen Raumbegriff nicht expliziert, lässt sich ganz entlang Giddens' Modell der Trennung von Raum und Ort begreifen, die durch die Arbeit der Statistik an der Wirklichkeit noch in Verbindung stehen. Kann sich aber dieser mathematisch-diagrammatische Raum von der gleichsam körperlichen Subjektivität (und damit von örtlicher Präsenz) als eigenständiger Raum de facto lösen? Anhand der Entwicklung der Meinungsforschung, die im gewissen Sinne das Quasi-Monopol der >öffentlichen Meinung übernimmt ${ }^{27}$ und damit nichts anderes als die Fortsetzung der Logik der Statistik auf das Sprechen des Souveräns selbst darstellt (und nicht mehr nur auf die Existenz der Körper selbst), lässt sich dieses Spannungsfeld von verschiedenen Räumen, im Hinblick auf wissenstechnische Realitäten, genauer betrachten.

\section{Die Verdatung der öffentlichen Meinung}

Hinsichtlich der Geschichte sozialwissenschaftlichen Wissens lässt sich die Einführung der Meinungsforschung unschwer als ein Projekt der Sozialforschung begreifen, eine >Geometrie des Heterogenen` zu etablieren, in der die >Unübersichtlichkeitく, die die große Gesellschaft produziert, durch mathematische Durchdringung der Vielfalt wieder in eine bestimmbare Ordnung gebracht wird. ${ }^{28}$ Gleichzeitig aber ist das Unterfangen der politischen Meinungsforschung ein Besonderes: Es schafft nicht einen Raum über neue Wissenstechniken, sondern das neue Wissen siedelt sich gerade an einer Bruchstelle zwischen Orten des Politischen und seiner Neuerschaffung als politischer Raum an: Das Bild des zum Publikum versammelten Souveräns und die Eigenlogik mathematischer Formen lassen sich erneut schwerlich zusammenbringen. Doch ist die Flexibilität des Instrumentariums und die Verbreitung dieser Industrie so umfassend, dass durch die schiere Menge der erzeugten Evidenzen der Eindruck der Integration von Allem und Jedem entstehen mag. Die Anschlussfähigkeit des neuen Wissens an bestehende Konstrukte ergibt sich dabei über das Konzept der >öffentlichen Meinung`, getragen von einem weiteren Singular, dem

$27 \mathrm{Vgl}$. Loic Blondiaux: La fabrique de l'opinion. Une histoire sociale des sondages, Paris: Seuil 1998, S. 9

28 Vgl. zur Diskussion dieses Projekts der >Geometrie des Heterogenen Anfang der Sozialwissenschaft Peter-Ulrich Merz-Benz' exemplarische Studie zum Werk Tönnies': Tiefsinn und Scharfsinn. Ferdinand Tönnies' begriffliche Konstitution der Sozialwelt, Frankfurt/Main: Suhrkamp 1995, bes. S. $108 \mathrm{ff}$. des $>$ Publikums $\measuredangle$, die zusammen ein fiktives Substrat, die Öffentlichkeit, bilden. ${ }^{29}$

Gerade der per se mathematisch definierte politische Raum begünstigte wohl die Quantifizierbarkeit dieser Konstrukte: Über einen zeitlichen Rhythmus, nämlich jenen der Wahlen, konstituiert sich der politische Raum immer von Neuem. Die Urne, welche zu den Wahlbüros transportiert wird, verkörpert das materiale Medium, das Ort (über den Akt des Wählens im Lokal) und Raum (die Mathematik der Verteilung der Stimmen) verbindet. ${ }^{30}$ Auch die Meinungsforschung pries sich als revolutionärer wissenschaftlicher Durchbruch, um die Orte des politischen Sprechens mit dem mathematisch konstituierten Souverän wieder in Einklang zu bringen. Sie pries sich an als Technologie, den politischen Raum mit neuem Wissen zu versehen.

1935 hatte sich in der Washington Post die erste Meinungsforschung der modernen Art der Öffentlichkeit mit diesem Versprechen präsentiert, und es lohnt sich, auf die Merkmale zu achten, mit denen sich die Meinungsforschung in den politischen Diskurs einführte (vgl. Abbildung 1).

Der Souverän erhält eine neue Stimme, eine neue Artikulationsform als Singular, so lautet die primäre Botschaft: »America Speaks«. Die Inklusionsproblematik des politischen Systems wird als behoben betrachtet, indem explizit jene Bevölkerungsteile gesucht werden, die sonst vom politischen Diskurs ausgeschlossen sind, aber nunmehr eine Stimme erhalten: »Does the Forgotten Man Still Feel Forgotten? He Answers for Himself in the Weekly Poll.«

Jenseits dieses Versprechens ist es jedoch erstaunlich, wie wenig sich die ikonischen Elemente der Meinungsforschung seit ihrem ersten Auftreten gewandelt haben: Sowohl diskursive wie nicht-diskursive Elemente der Darstellung der öffentlichen Meinung sind beinahe identisch geblieben, ungeachtet dessen, dass die zugrunde liegenden Erhebungsverfahren

29 „Öffentlichkeit war das Medium, durch das und innerhalb dessen sich das Volk als politischer Körper konstituierte« (Lucian Hölscher: »Öffentlichkeit«, in: Karlfried Gründer/Joachim Ritter (Hg.), Grundbegriffe der Philosophie, Bd. 4, Basel, Stuttgart: Klett 1978, S. 413-467, hier S. 456).

30 Die schiere Größe der Bevölkerung in den USA, die Heterogenität der Bevölkerung, im Gleichzug mit massenmedialer Fragmentierung, führten dazu, dass die Konstitutionsmöglichkeiten des politischen Raums und damit die Aktionsmöglichkeiten des konstituierten Souveräns angesichts der Intensität politischer Kommunikationen immer wieder nicht gelingt. Die Orte des politischen Sprechens und die Konstitution des Raumes gerieten in Ungleichgewicht. Diese besonderen Umstände begünstigten die Durchsetzung standardisierender, quantifizierender Techniken: Noch früher als in der politischen Meinungsforschung zeigten sie sich in der Marktforschung (vgl. Susan Strasser: Satisfaction Guaranteed. The Making of the American Mass Market, New York: Bass 1989). 
sich in technischer Hinsicht (nicht aber in methodologischer) auf vielfältige Weise entlang neuer Kommunikationstechnologien veränderten. Gerade diese ikonischen i.e. vornehmlich diagrammatischen Elemente zeigten indes, auf welche Weise die Meinungsforschung den politischen Raum der Artikulation des Souveräns auf technische Weise neu erschafft. Die >Figur des Publikums` erhält nun einen eminent diagrammatischen Charakter. Eine Grafik berichtet in gewohnter Weise über einen »Trend of Opinion«: Untereinander aufgeführt sind drei verschiedene Balkendiagramme, welche die Einstellungen zur Sozialfürsorge erfassen.

Unten findet sich eine Karte der USA, in der Werte zu denselben Fragen eingetragen sind. Das untere Diagramm folgt damit noch der Logik der Geometrisierung des Souveräns, wie sie die amerikanische Verfassung vorschrieb: ein klassisches Tableau des Wissens im Sinne Foucaults, gebildet aus Mathesis und Taxonomia. ${ }^{31}$ In besonderer Weise stellt aber das obere Diagramm ein spezifisches Merkmal des Wissens der Meinungsforschung dar; die zeitliche Abstufung der Bewegung einer vermessenen Einstellung. Damit wird jenseits des institutionellen Rhythmus' der Wahlen der politische Raum konstituiert. Die >Vermessung der >öffentlichen Meinung ist $\mathrm{zu}$ einem beliebigen Zeitpunkt möglich. Damit wird die Zeit fließend, aber diagrammatisch in einem neuartigen symbolischen Zeit-Raum aufgehoben. Die geometrischen Räume reihen sich in diachroner Reihe aneinander, ergeben aber als Ganzes eine synchrone Entität, welche die zeitlichen Begrenzungen der Zustände des politischen Raumes aufhebt. Hierin finden sich aber gerade wiederum die Mechanismen der Loslösung eines abstrakten Raumes vom Ort über das Prinzip der Verzeitlichung, das ein neues symbolisches Medium ermöglicht: Ernest Renans plébiscite de tous les jours wird in einem virtuellen, mathematischen Raum möglich. Mehr noch, mit den flexiblen Techniken, die versprechen, sämtliche Orte des Politischen zu erreichen, sofern notwendig, wird dem Versprechen nach die Paradoxie der Inklusion des Publikums im politischen Raum gelöst. Wo immer politisches Sprechen ist, lässt es sich prinzipiell erfassen und im Raum des Politischen als Singular darstellen: Der Souverän spricht im Medium der Diagramme der Meinungsforschung. Alle Unzulänglichkeiten, die etwa bei einem direkten Vergleich mit Wahlen zum Vorschein treten, sind dabei bis heute nur Ausdruck behebbarer methodisch-technischer Mängel. ${ }^{32}$ Doch sind damit die

31 Vgl. Keller: Archäologie der Meinungsforschung (wie Anm. 4), Kap. 7.

32 Die Argumente, die gegen eine Quantifizierung des Konstrukts $>$ Meinung eingewandt wurden, sind in Keller: Archäologie der Meinungsforschung (wie Anm. 4) aufgeführt. Zu einer konzisen Darlegung vgl. auch Pierre Bourdieus Aufsatz "Die öffentliche Meinung gibt es nicht $\ll$, in: ders., Soziologische Fragen, Frankfurt/Main: Suhrkamp 1993, S. 212-223.
Abb. 1: Die Präsentation der ersten Meinungsforschungsergebnisse in der Öffentlichkeit (Washington Post vom 20. Oktober 1935)

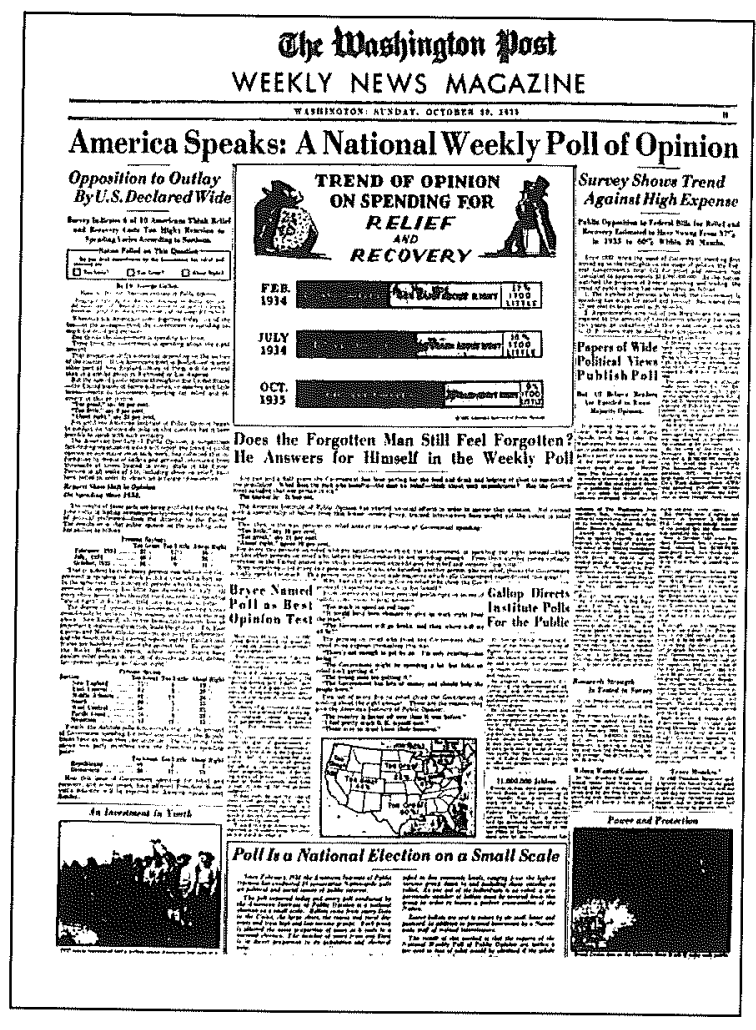

Quelle: Normand Bradburn/Seymour Sudman: Polls and Surveys. Understanding What They Tell Us, San Francisco, London: Bass 1988, S. 20-21.

Aporien der Inklusion in den politischen Raum wirklich aufgehoben oder bloß auf eine andere Ebene verschoben?

Das Problem der Meinungsforschung siedelt sich exakt in der Aporie an, vor der Hayter stand, nur natürlich auf einer ganz anderen Ebene. Auf welche Weise können die politischen Subjekte, welche das Publikum, den Souverän formieren, in einen mathematisch-proportionalen Raum überführt werden, der qua seiner geometrischen Ordnung gerade nicht dem Singulären der politischen Rede folgt? Auf welche Weise lassen sich die Individuen als sprechende Subjekte mit eigenen Interpretationshorizonten porträtieren in einem höchst standardisierten Verfahren? Gallup war sich der Problematik - vielleicht mehr noch als heutige Apologeten des Verfahrens - durchaus bewusst. Gerade die Medialität der Vermittlung von 
Bevölkerung und politischen Institutionen ist für ihn Signum der Moderne. ${ }^{33}$ Seine Strategie, seine Utopie war, den diagrammatischen Raum, den er sich eröffnete, wieder auf einen ursprünglichen Schauplatz der Politik zurückzuführen, ein fugenloses Übergehen von den Orten des Sprechens zum politischen Raum, und nicht ein qualitativ Anderes seiner Techniken zu postulieren.

Gallup argumentiert sehr ausgeprägt mit den Realitäten großer technischer Gesellschaften, die er zur Imagination eines ursprünglich lokalen Ortes des Politischen setzt und hierzu die politische Theorie von James Bryce zur Begründung heranzieht. ${ }^{34}$ Diesen Ort ursprünglichen Politisierens verkörpert für Gallup das New England Town Meeting, Bürgerversammlungen lokaler Towns - ähnlich der Gemeindeversammlung in der Schweiz - zwecks Beratung von Verwaltungs- und Regierungsgeschäften. Diese Orte sind für ihn Modell der demokratischen Politik, die es aber an die Realitäten der grossen Gesellschaften anzupassen gilt, die sich durch Zahl der Bevölkerung ebenso wie durch neue Kommunikationstechnologien auszeichnet. Die Versöhnung von politischem Raum und Schauplatz des Politischen, respektive der Versuch der Wiedererschaffung des Ortes über den diagrammatischen Raum, wird in folgendem Zitat einer Rede, die Gallup vor der Baker-Foundation hielt, deutlich. Die Verbindung zwischen neuen Technologien, traditionellen Medien und Meinungsforschung bedeute,

$\gg[\ldots]$ that the nation is literally in one great room. The newspaper and the radio conduct the debate on national issues, presenting both information and argument on both sides, just as the townsfolk did in person in the old form meeting. And finally, through the process of the sampling referendum, the people, having heard the debate on both sides of every issue, can express their will. After one hundred and fifty years we return to the town meeting. This time the whole nation is within the door. $\aleph^{35}$

33 »The enormous expansion of social and political life broke down the old face to face relationships of the small governing class [...]. The impetus of growing industrialism, the revolutions of transport and communication, the emergence of factories, towns, and great cities destroyed for all times the rural localism of early America.« (George Gallup/Saul Forbes Rae: The Pulse of Democracy. The Public-Opinion Poll and How It Works, New York: Simon und Schuster 1940, S. 13f.).

34 Vgl. James Bryce: The American Commonwealth, New York: MacMillan 1919; ders.: Modern Democracies, London: MacMillan 1921.

35 George Gallup: Public Opinion in a Democracy, Princeton: Baker Foundation 1939, S. 15
Der eine grosse Raum der Nation, der Konstitutionsort des Souveräns, wird durch die Technologie der Meinungsforschung neu errichtet, mit dem Versprechen der umfassenden Inklusion (»This time the whole nation is within the door.(). Dass dieser Raum mit Sinn erfüllt wird, dafür leistet der Rückbezug auf den Ort unmittelbarer politischer Rede Gewähr. Es ist der Interviewer, der als intermediäre Instanz zwischen Ort und Raum fungiert. Als Wissensvermittler bleibt er aber als Person gleichsam körperlich im Ort verhaftet, was Gallup beinahe dramatisch zum Ausdruck bringt, wie folgende Stelle aus seinem Werk mit dem Namen The Pulse of Democracy exemplarisch zeigt:

»These interviewers [des American Institute of Public Opinion] know what it is to drive trough a Maine snowstorm to make a farm interview; to trudge across Kansas wheat fields on a blistering day to interview a tresher on the job; to travel trough the red-clay mud of Georgia in a drenching rainstorm «. ${ }^{36}$

Doch nicht nur die Natur macht dem Interviewer zu schaffen, sondern auch die verruchten Gegenden der New Yorker East Side, die ebenso unentwegt erforscht werden wie die von Schneestürmen überzogenen Landschaften.

Inwiefern zeigen sich die angesprochenen Paradoxien der umfassenden Integration des Publikums auch in der Meinungsforschung? Hayter ist daran gescheitert, die Mitglieder des Parlaments in ihrer Subjektivität zu porttätieren und gleichzeitig die große Menge der Parlamentarier in dem Bild unterzubringen. In ähnlicher Weise stellt sich das Problem der Meinungsforschung, aber nicht über das Medium des Bildes, sondern über dasjenige der Sprache als Medium der politischen Subjektivität. Auf welche Weise lassen sich die Wörter - in standardisierten Befragungen sogar vorgegebene Wörter wie $>$ Ja $\prec$ und $>$ Nein $\prec$ - als Ausdruck einer subjektiven Position deuten?

Dieses Problem, das notabene in diagrammatischen Darstellungen gar nicht erscheinen kann, so tiefgründig die methodischen Reflexionen bei der Erzeugung solcher Ergebnisse auch sein mögen, hat unversehens Kritik hervorgerufen; eine Kritik, die bis heute an den Ergebnissen der Meinungsforschung geäußert wird. ${ }^{37}$ Lindsay Rogers hatte ihr bereits 1949 den Weg gewiesen. ${ }^{38}$ Die Aufsummierung von yeses und noes habe nichts

36 Gallup/Rae: The Pulse of Democracy (wie Anm. 33), S. 4.

$37 \mathrm{Vgl}$. in konziser Weise: Pierre Bourdieu: $s$ Meinungsforschung - Eine, Wissenschaft ohne Wissenschaftler«, in: ders. (Hg.), Rede und Antwort, Frankfurt/Main: Suhrkamp 1992, S. 208-216; ausführlich: Aaron V. Cicourel: Methode und Messung in der Soziologie, Frankfurt/Main: Suhrkamp 1974.

38 Vgl. Lindsay Rogers: The Pollsters. Public Opinion, Politics, and Democratic Leadership, New York: Knopf 1949. 
mit einer politischen Debatte zu tun, die letztendlich immer einen Prozess darstelle, der aber im Zustand des Erstarrtseins in Zahlen nicht zum Ausdruck komme. Rogers kappt damit explizit die versuchte Verbindung des diagrammatischen Raumes. Das bloße Zählen von Stimmen habe bereits in Disputen politischer Versammlungen keinen Sinn ergeben: »[N]aked numerical decision is meaningless $«,{ }^{39}$ da das Ziel eines beratenden politischen Publikums letztlich sei, wenn möglich Einigung zu erreichen oder zumindest Kompromisse zu finden und nicht numerisch Mehrheiten zu erzielen:

"In the New England town meetings, that Dr. Gallup views with nostalgia, there could be argument for and against a proposition at the same time in the presence of all who were to vote, and the proposition under debate could be reframed to meet objections that the discussion had brought out. It was rare that in the town meeting a small majority lorded it over a substantial minority. Usually objections could be met, and the decisions could have a wide basis of assent. Only extremists were left in opposition. ( $^{40}$

Wiederum äußert sich in dieser Kritik dasselbe Spannungsfeld von Raum und Prozess: Die Fixierung des Politischen in einem geometrischen Raum scheitert an der Zeitlichkeit des politischen Diskurses und der nicht reduzierbaren Subjektivität der am Diskurs Beteiligten selbst. Wie bei Arendt, so zeigt Rogers Kritik die de facto vollzogene Trennung von Ort und Raum im Bereich der Demokratien, die scheinbar unabänderliche Eigenlogik des symbolisch-mathematischen Raumes der Erzeugung von Bildern des Souveräns. Bislang ist keine Technik bekannt, die in diesem Ausmaß so umfassende Repräsentationen des politischen Publikums zu produzieren vermöchte. ${ }^{41}$ Ein grundsätzliches Scheitern, wie es in Hayters Bild offenbar wird, scheint kaum mehr möglich, eine Politik ohne Meinungsforschung liegt im Bereich des Unvorstellbaren. ${ }^{42}$ Angesichts der schieren Menge von erzeugten diagrammatischen Repräsentationen des Souveräns über die alltäglich präsentierten Meinungsforschungsergebnisse treten solche Paradoxien fast vollständig in den Hintergrund.

39 Ebd., S. 72

40 Ebd., S. 71

41 Vgl. Patrick Champagne: Faire l'opinion. Le nouveau jeu politique, Paris: Minuit 1990; Blondiaux: La fabrique de l'opinion (wie Anm. 27).

42 Vgl. Neidhardt: „Öffentlichkeit, öffentliche Meinung, soziale Bewegung« (wie Anm. 26). 